\title{
YAP and Wnt3a independently promote AECIIs proliferation and differentiation by increasing nuclear $\beta$-catenin expression in experimental bronchopulmonary dysplasia
}

\author{
XIANXIAN JIA, BO WU, JINHUI HUANG, LIN FAN, MIAO YANG and WEI XU \\ Department of Pediatrics, Shengjing Hospital of China Medical University, Shenyang, Liaoning 110004, P.R. China
}

Received August 4, 2020; Accepted October 27, 2020

DOI: $10.3892 / \mathrm{ijmm} .2020 .4791$

\begin{abstract}
Arrested alveolar development is the main pathological characteristic of neonatal bronchopulmonary dysplasia (BPD); however, a number of studies aiming to improve alveolarization have focused on alveolar epithelial cell damage and impairment. Previously, the authors reported that the Wnt signaling plays a key role in alveolar injury and repair by regulating alveolar epithelial type II cell (AECII) proliferation and differentiation. In the present study, the authors wished to investigate whether Yes-associated protein (YAP), a transcriptional coactivator in the Hippo signaling pathway, affects AECII proliferation and differentiation via the $\mathrm{Wnt} / \beta$-catenin pathway in BPD. It was found that YAP regulated AECII proliferation and differentiation. A decreased expression of YAP, Wnt3a and nuclear $\beta$-catenin was observed in lung tissues affected by BPD. In vitro, YAP and Wnt3a overexpression in BPD promoted AECII proliferation and differentiation into AECIs by increasing the nuclear transfer of $\beta$-catenin and vice versa. The effects of a decreased Wnt3a expression in primary AECIIs in BPD were compensated by YAP overexpression, as were the effects of Wnt3a knockdown in primary AECIIs. On the whole, the findings of the present study demonstrate that YAP and Wnt3a independently promote AECII proliferation and differentiation in experimental BPD by increasing the nuclear $\beta$-catenin levels. Therefore, Wnt3a or YAP may be candidate regulatory targets for improving the outcomes of BPD.
\end{abstract}

\section{Introduction}

Bronchopulmonary dysplasia (BPD), a severe lung dysfunction, is common in premature infants born at a gestational age of $<29$ weeks, when lungs are in the cystic development

Correspondence to: Professor Wei Xu, Department of Pediatrics, Shengjing Hospital of China Medical University, 36 Sanhao, Shenyang, Liaoning 110004, P.R. China

E-mail: tomxu.123@163.com

Key words: Yes-associated protein, proliferation, differentiation, alveolar epithelial type II cells, Wnt/ $\beta$-catenin stage (1). The incidence of BPD can be as high as $40 \%$, particularly in infants that weigh $<1,500 \mathrm{~g}$ (2). Moreover, BPD affects the survival and quality of life of premature infants (3). The majority of these infants require long-term oxygen therapy to maintain lung function (4). Alveoli are gas-exchange sacs lined by cuboidal shaped squamous alveolar epithelial type I cells (AECIs) and alveolar epithelial type II cells (AECIIs), which secrete active substances on their surface (5). AECIIs are widely referred to as 'alveolar stem cells' and contribute toward lung repair and regeneration (5). Indeed, studies have demonstrated that the proliferation and differentiation of AECIIs into AECIs plays a key role in the repair of damaged alveoli $(6,7)$. Since arrested alveolar development is the main pathological feature of neonatal BPD, a number of studies have investigated alveolar epithelial cell damage and repair to improve alveolarization (8-10) and enhance the understanding of the mechanisms of alveolar epithelial cell regeneration in order to develop treatment methods for BPD.

Yes-associated protein (YAP) is a key downstream effector of the Hippo pathway $(11,12)$, and recent studies have demonstrated that YAP exerts regulatory effects on a number of stem cells (13-15). However, the role of YAP in the proliferation and differentiation of AECIIs into AECIs remains controversial (16-19). For instance, Sun et al reported that although YAP is not involved in these processes, TAZ plays a critical role in AECIIs to AECIs differentiation and maintaining alveolar integrity following injury (20); however, Seo et al found that YAP/TAZ contributed towards alveolar epithelial regeneration (21). Thus, the regulatory role of YAP in the proliferation and differentiation of AECIIs is likely complex and requires further investigation.

Previously, the authors reported that Wnt signaling plays key roles in alveolar injury and repair by regulating the proliferation and differentiation of AECIIs $(22,23)$. The Wnt signaling pathway consists of both canonical and noncanonical pathways (24) involving Wnt proteins, receptors and regulatory factors that control numerous biological processes, including cell proliferation, development and differentiation $(25,26)$. Wnt3a signals via the canonical pathway and is expressed in bronchial and alveolar epithelial cells; however, it exhibits an increased expression in fibrotic regions and the hypertrophic alveolar epithelium (27). Several studies have reported conflicting associations between YAP and the Wnt/ $\beta$-catenin signaling pathway $(19-21,28,29)$. For instance, 
Imajo et al reported that the Hippo pathway effectors, YAP and TAZ, bound to $\beta$-catenin and retained it in the cytoplasm to suppress $W n t / \beta$-catenin signaling from $293 \mathrm{~T}$ cells (29), whereas Jia et al found that YAP promoted the osteogenic differentiation of human periodontal ligament stem cells in vitro and inhibited adipogenic differentiation via low-density lipoprotein receptor-related protein 6 (LRP6) and dishevelled segment polarity protein 3 (DVL3)-mediated Wnt/ $\beta$-catenin signaling (28). Therefore, it remains unclear whether YAP affects the proliferation and differentiation of AECIIs via the $\mathrm{Wnt} / \beta$-catenin pathway in BPD.

The present study thus aimed to determine the role of YAP in the proliferation and differentiation of AECIIs and the involvement of the $\mathrm{Wnt} / \beta$-catenin pathway in this process in BPD. It was demonstrated that YAP expression was decreased in experimental BPD and played a key role in promoting the proliferation and differentiation of AECIIs, consistent with a previous study by the authors on Wnt3a (23). In addition, it was found that Wnt3a overexpression could compensate for YAP depletion and vice versa; however, neither altered the expression of the other. Moreover, neither YAP nor Wnt3a overexpression increased total $\beta$-catenin expression in AECIIs in vitro, but both increased nuclear $\beta$-catenin levels in these cells. Thus, a functional association was identified between YAP and Wnt/ $\beta$-catenin signaling. This indicates that Wnt3a and YAP cooperatively regulate the proliferation and differentiation of AECIIs in experimental BPD.

\section{Materials and methods}

Animal models. All animal experiments were examined and approved by the Experimental Animal Ethics Committee of China Medical University (2017PS086K). The BPD model in the present study has been previously described $(22,30)$. In brief, 20 adult Sprague-Dawley (SD) rats were purchased from Liaoning Changsheng Biotechnology, and females and males were mated at a ratio of $4: 1$. Feeding was carried out in a temperature-controlled room during a 12-h light/dark cycle, with a temperature of $23 \pm 2^{\circ} \mathrm{C}$ and a relative humidity of $40-60 \%$. Each pregnant rat was fed independently until natural delivery on days 21-23. Within $12 \mathrm{~h}$ of delivery, the neonatal and maternal rats were randomly divided into the BPD model or control groups that were exposed to $80-85 \%$ oxygen in a sealed plexiglas tank for 1-21 days or room air ( $21 \%$ oxygen), respectively. The number of rats in each animal model preparation was assigned as follows: In total, 3 cages (1 maternal and 12-13 newborn rats in each cage) were placed in an oxygen chamber as the BPD model group, and another 3 cages (1 maternal and 12-13 newborn rats in each cage) were placed in the room with normal air as the control group. The oxygen concentration was monitored continuously using an oxygen meter. The box was opened regularly for 30 min each day and maternal rats were switched between the model and control groups every $24 \mathrm{~h}$ to prevent oxygen toxicity.

Lung tissue specimens and lung histology. On postnatal days 1, $3,7,14$ and 21,10 neonatal rats from each group were randomly selected and all rats were anesthetized by an intraperitoneal injection of $1 \%$ pentobarbital sodium $(35 \mathrm{mg} / \mathrm{kg})$. Under aseptic conditions, the left auricle was reduced, the right ventricle was per-fused with PBS and lung tissues were collected. The lower lobe of the right lung was fixed in $4 \%$ para-formaldehyde (PFA) and 3- $\mu \mathrm{m}$-thick sections were prepared for hematoxylin and eosin (H\&E), immunohistochemical (IHC) and immunofluorescence (IF) staining. The remaining lung lobes were preserved at $-80^{\circ} \mathrm{C}$ for use in subsequent analyses. Lung tissue sections (3- $\mu \mathrm{m}$-thick) were deparaffinized in xylene for $20 \mathrm{~min}$ and rehydrated in 100, 95, 85 and $70 \%$ alcohol for $10 \mathrm{~min}$ before $\mathrm{H} \& \mathrm{E}$ staining was performed. Briefly, specimens were stained with hematoxylin for $2 \mathrm{~min}$ at $37^{\circ} \mathrm{C}$, rinsed, and blued for nuclear staining under running water for $15 \mathrm{~min}$. Sections were then stained with eosin for $1 \mathrm{~min}$ at $37^{\circ} \mathrm{C}$, dehydrated using 70,95 and $100 \%$ alcohol, made transparent using xylene, and embedded in neutral resin. Under a microscope (Nikon Corporation), by drawing a perpendicular line from the center of the most peripheral bronchiole to the pleura or the nearest interlobular septum, the number of alveoli transected by this line was defined as the radial alveolar count (RAC), an important index used to evaluate the stage of lung development (31). Image $\mathrm{J}$ software version 1.51 was used to measure alveolar wall thickness.

Isolation of AECIIs. AECIIs from the neonatal rats were isolated, purified and cultured as previously described (22). Following cardiopulmonary lavage, the cut lung tissue was digested in $0.25 \%$ trypsin-EDTA (25200056, Gibco; Thermo Fisher Scientific, Inc.) for $30 \mathrm{~min}$ and then digestion was terminated using the same volume of DMEM/F12 medium (SH30023.01B, Gibco; Thermo Fisher Scientific, Inc.) containing 10\% fetal bovine serum (10099141, Gibco; Thermo Fisher Scientific, Inc.). The disassociated cells were filtered through a 200 -mesh cell strainer, centrifuged $\left(4^{\circ} \mathrm{C}\right.$, $5 \mathrm{~min}$ and $200 \mathrm{x} \mathrm{g}$ ) and the cells were then digested using $0.1 \%$ type I collagenase (MB2686, Meilunbio) for $20 \mathrm{~min}$. The cells were harvested by centrifugation $\left(4^{\circ} \mathrm{C}, 5 \mathrm{~min}\right.$ and $200 \mathrm{x} \mathrm{g}$ ), resuspended in DMEM/F12 medium containing $1 \%$ penicillin streptomycin double antibody (Gibco; Thermo Fisher Scientific, Inc.) and 10\% fetal bovine serum, and incubated in a $5 \% \mathrm{CO}_{2}$ incubator at $37^{\circ} \mathrm{C}$. Fibroblasts were removed using the differential attachment procedure (repeated 3 times, $1 \mathrm{~h}$ each) and purified. Following $24 \mathrm{~h}$ of culture, the activity $(>95 \%)$ and purity $(>90 \%)$ of AECIIs were measured using trypan blue staining for $3 \mathrm{~min}$ at $37^{\circ} \mathrm{C}$ (C0011-1, Beyotime Institute of Biotechnology, Inc.) and AECII-specific labeling of surfactant protein C (SPC) immunostaining, respectively.

IHC staining. Prior to IHC staining, the sections were deparaffinized, microwave-heated $\left(98-100^{\circ} \mathrm{C}\right)$, and treated sequentially with $3 \% \mathrm{H}_{2} \mathrm{O}_{2}$ for $20 \mathrm{~min}$ and goat serum for $1 \mathrm{~h}$ at $37^{\circ} \mathrm{C}$. The sections were then incubated with the following primary antibodies diluted in $\mathrm{PBS}$ at $4^{\circ} \mathrm{C}$ overnight: Anti-YAP (\#14074, 1:400, Cell Signaling Technology, Inc.) and anti- $\beta$-catenin (WL0962a, 1:100, Wanleibio, Co., Ltd.). The sections were then incubated with secondary antibodies (goat anti-rabbit/mouse, kit9710, MXB Biotechnologies) at $37^{\circ} \mathrm{C}$ and streptavidin-horseradish peroxidase for $20 \mathrm{~min}$ at $37^{\circ} \mathrm{C}$, developed with $3,3^{\prime}$-diaminobenzidine, and counterstained with hematoxylin for $2 \mathrm{~min}$ at $37^{\circ} \mathrm{C}$. Finally, the 
sections were observed under a light microscope (Nikon Corporation), where 6 fields of view were randomly selected for each sample.

IF staining. Prior to IF staining, the cells were fixed with $4 \%$ PFA for 15 min. After blocking with $10 \%$ goat serum (SL038, Beijing Solarbio Science \& Technology Co., Ltd.) at $37^{\circ} \mathrm{C}$ for $15 \mathrm{~min}$ and incubated with 4 combinations of 2 primary antibodies overnight at $4^{\circ} \mathrm{C}$ as follows: i) Rabbit polyclonal anti-SPC (PA5-71680, 1:40, Thermo Fisher Scientific, Inc.) and mouse monoclonal anti-YAP (66900-1-lg, 1:50, Proteintech Group, Inc.); ii) Rabbit polyclonal anti-SPC and mouse monoclonal anti- $\beta$-catenin (610154, 1:100, BD Biosciences); iii) Rabbit polyclonal anti-aquaporin 5 (AQP5; ab78486, 1:200, Abcam) and mouse monoclonal anti-YAP; iv) Rabbit polyclonal anti-AQP5 (ab78486, 1:200, Abcam) and mouse monoclonal anti- $\beta$-catenin. The sections were then incubated with goat anti-rabbit IgG (SA00007-2, 1:200, Proteintech Group, Inc.) and goat anti-mouse IgG (SA00003-1, 1:200, Proteintech Group, Inc.) secondary antibodies coupled to fluorescein isothiocyanate in the dark for $4 \mathrm{~h}$ at $37^{\circ} \mathrm{C}$, followed by 4',6-diamidino-2-phenylindole (DAPI, D820010, Beijing Solarbio Science \& Technology Co., Ltd.) to nuclear staining for $5 \mathrm{~min}$ at $37^{\circ} \mathrm{C}$. Double IF images were acquired using a confocal laser-scanning microscope (Nikon Corporation) and 6 fields of view were randomly selected for each sample.

Cell transfection. Primary cells obtained from BPD and control rats were inoculated in 6-well plates (a total of 200,000 cells were seeded into each well of the 6-well) and cultured to a cell density of approximately $60-70 \%$. While the cells in the control group were left untreated, the cells in the BPD group were transfected with either small interfering RNA (siRNA)-negative control (NC, GenePharma), siRNA-YAP (GenePharma), siRNA-Wnt3a (GenePharma), overexpression plasmid YAP (OEYAP, Genechem), overexpression plasmid Wnt3a (OEWnt3a, Genechem), or overexpression plasmid NC (OENC, Genechem). The siRNA sequences were as follows: siYAP sense, 5'-GAUUGAAACAGCAGGAGUUTT-3' and antisense, 5'-AACUCCUGCUGUUUCAAUCTT-3'; siWnt3a sense, 5'-GGUGGUCGCAGCCUGACUUTT-3' and antisense, 5'-AAGUCAGGCUGCGACCACCTT-3'; NC sense, 5'-UUC UCCGAACGUGUCACGUTT-3' and antisense, 5'-ACGUGA CACGUUCGGAGAATT-3'. At transfection in the 6-well plate, the amount of siRNA and plasmid was 7.5 and $2 \mu \mathrm{g}$ respectively, and the amount in the control group was the same. Transfection was performed using Lipofectamine 3000 (L3000015, Thermo Fisher Scientific, Inc.), according to the manufacturer's instructions and was confirmed by PCR and western blot analysis. After 48-72 h, cells were collected for further analysis.

Western blot analysis. Total proteins from the lung tissues and AECIIs were lysed using radio immunoprecipitation assay (RIPA) buffer (P0013B, Beyotime Institute of Biotechnology, Inc.) with $1 \%$ phenylmethanesulfonyl fluoride (PMSF; ST506, Beyotime Institute of Biotechnology, Inc.). Nuclear and cytoplasmic proteins were extracted using a Nuclear and Cytoplasmic Protein Extraction kit (20126ES50, Beyotime Institute of Biotechnology, Inc.) according to the manufacturer's instructions. The protein concentration was determined by the bicinchoninic acid (BCA; p0010, Beyotime Institute of Biotechnology, Inc.) method. An equal amount of protein $(40 \mu \mathrm{g})$ of each sample was used for western blotting. Proteins were then separated by $10 \%$ sodium dodecyl sulfate polyacrylamide gel electrophoresis (SDS-PAGE) and transferred onto polyvinylidene fluoride (PVDF) membranes. After blocking with $5 \%$ non-fat milk for $1 \mathrm{~h}$ at $37^{\circ} \mathrm{C}$, the membranes were incubated with the following primary antibodies diluted in PBS overnight at $4^{\circ} \mathrm{C}$ : Anti-YAP (66900-1-lg, 1:500, Proteintech Group, Inc., $65 \mathrm{kDa})$, anti-Wnt3a (DF6113, 1:1,000, Affinity, $39 \mathrm{kDa}$ ), anti-SPC (PA5-71680, 1:2,000, Thermo Fisher Scientific, Inc., $21 \mathrm{kDa}$ ), anti-AQP5 (ab78486, 1:5,000, Abcam, $24 \mathrm{kDa})$, anti- $\beta$-catenin (610154, 1:2,000, BD Biosciences, 92 kDa), anti-GAPDH (10494-1-AP, 1:5,000, Proteintech Group, Inc., $36 \mathrm{kDa})$ and anti-Lamin A (1:1,000, ab26300, Abcam, $74 \mathrm{kDa}$ ). Following incubation with goat anti-mouse IgG secondary antibodies (SA00001-1, 1:5,000, Proteintech Group, Inc.) or goat anti-rabbit IgG secondary antibodies (SA00001-2, 1:5,000, Proteintech Group, Inc.) at $37^{\circ} \mathrm{C}$ for $2 \mathrm{~h}$, signals were detected by enhanced chemiluminescence (34577, Thermo Fisher Scientific, Inc.). The gray values of the protein bands were analyzed using Image $\mathbf{J}$ software version 1.51 .

Reverse transcription-quantitative PCR (RT-qPCR). Total RNA was extracted from the lung tissues or AECIIs using TRIzol reagent (9108, Takara Bio, Inc.) and the samples were all treated with RNA DNase before the reverse transcription process according to the official instruction, and $1 \mu \mathrm{g}$ per sample was then reverse transcribed into cDNA using the PrimeScript ${ }^{\mathrm{RT}}$ reagent kit with gDNA Eraser (RR047A, Takara Bio, Inc.). Each targeted cDNA (2 $\mu \mathrm{l})$ was amplified using the TB Green PCR Core kit (RR820A, Takara Bio, Inc.) via the ABI 7500 system, and the following primers: SPC forward, 5'-GTG GTTGTGGTGGTAGTCCTTGTC-3' and reverse, 5'-CGA TGCCAGTGGAGCCAATAGAG-3'; AQP5 forward, 5'-CAA CAACACAACGCCTGGCAAG-3' and reverse, 5'-AGAGTC GGTGGAGGAGAAGATGC-3'; YAP forward, 5'-GCCATG AACCAGAGGATCACTCAG-3' and reverse, 5'-AGCCTC TCCTTCTCCATCTGTAGC-3'; $\beta$-catenin forward, 5'-GTT GCTCCACTCCAGGAATGAAGG-3' and reverse, 5'-GCA CCAATGTCCAGTCCGAGATC-3'; Wnt3a forward, 5'-TGG TGGTGGTGGTGGCAGAG-3' and reverse, 5'-CACAGC CAAGGACCAGAGAAGAAC-3'; GAPDH forward, 5'-GAC ATGCCGCCTGGAGAAAC-3' and reverse, 5'-AGCCCA GGATGCCCTTTAGT-3'. The following cycling conditions were used: $95^{\circ} \mathrm{C}$ for $30 \mathrm{sec} ; 40$ cycles of $95^{\circ} \mathrm{C}$ for $5 \mathrm{sec}$ and $60^{\circ} \mathrm{C}$ for $34 \mathrm{sec} ; 95^{\circ} \mathrm{C}$ for $15 \mathrm{sec} ; 60^{\circ} \mathrm{C}$ for $1 \mathrm{~min}$; and $95^{\circ} \mathrm{C}$ for $15 \mathrm{sec}$. To determine relative gene expression, target mRNA expression was calculated relative to GAPDH using the $2^{-\Delta \Delta \mathrm{Cq}}$ method (32).

Statistical analysis. Graphpad prism 5 was used for statistical analysis. All experiments involved at least 6 rats and were performed in triplicate. Results were expressed as the means \pm standard deviation (SD). All raw data were analyzed by one-way or two-way analysis of variance (ANOVA) followed by the Bonferroni test for post hoc comparisons. P-values of $<0.05$ were considered to indicate statistically significant differences. 
A

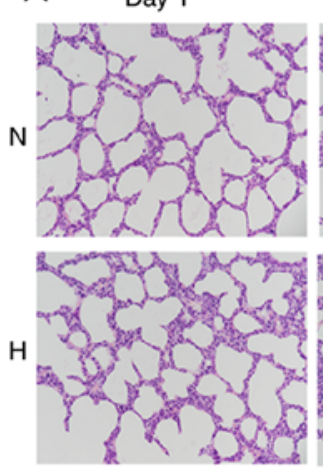

$\mathrm{B}$

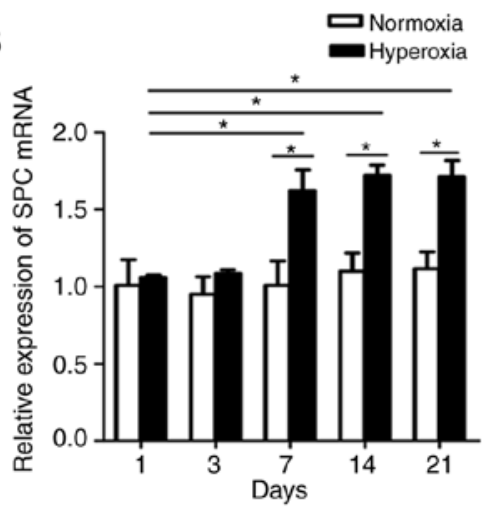

Day 7

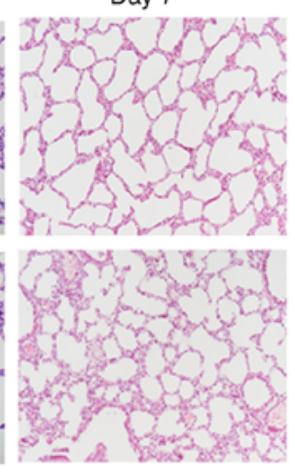

C
Day 14

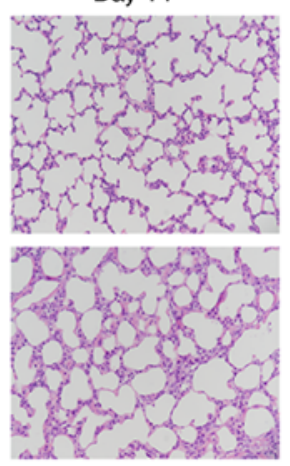

Day 21

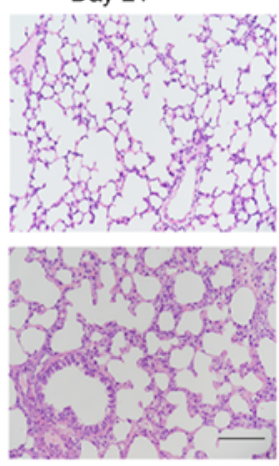

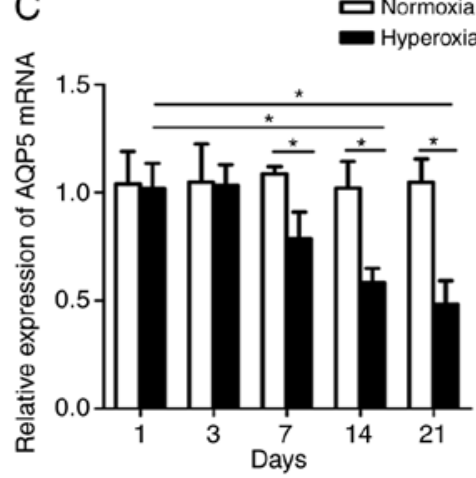

$$
\text { ㅁormoxia }
$$
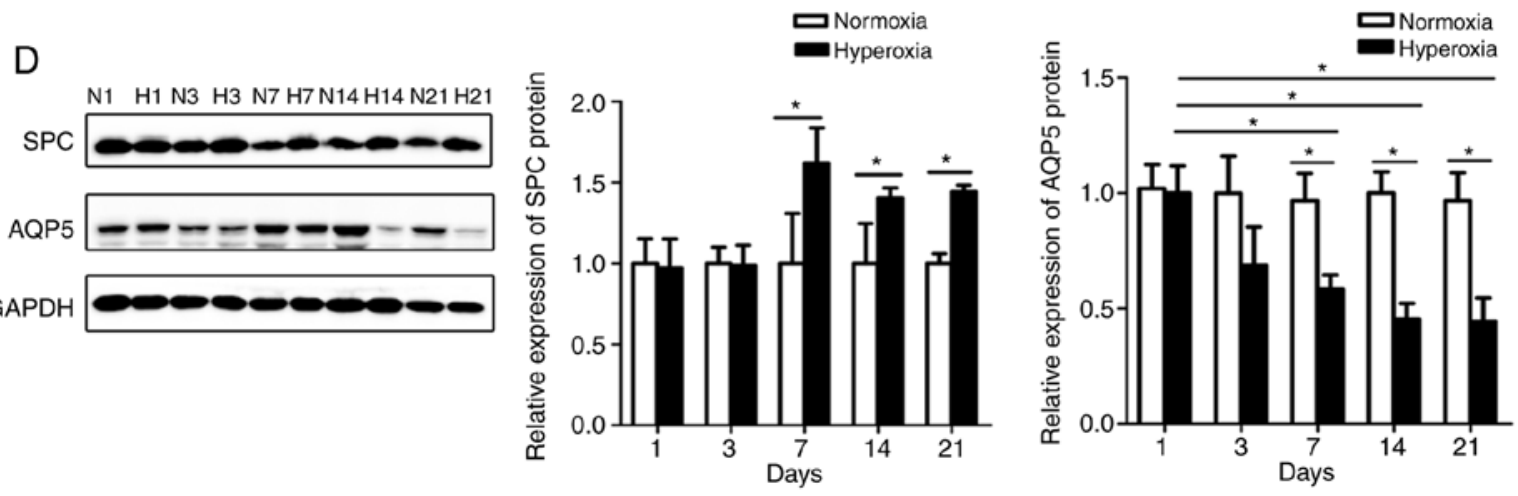

Figure 1. Hyperoxia exposure inhibits the differentiation of AECIIs to AECIs in vivo. (A) Hyperoxia exposure induces alveolar damage in premature rats (B-D) Hyperoxia increased (B) SPC mRNA and (D) protein expression at days 7, 14 and 21, and decreased (C) AQP5 mRNA and (D) protein expression at days 7, 14 and 21. Data are expressed as the means $\pm \mathrm{SD}(\mathrm{n}=6)$. ${ }^{*} \mathrm{P}<0.05$. Scale bar, $40 \mu \mathrm{m}$. N, normoxia-exposed rats; H, hyperoxia-exposed rats; AECIIs and AECIs, alveolar epithelial type II and I cells; SPC, surfactant protein C; AQP5, aquaporin 5.

\section{Results}

Experimental BPD is established by the induction of hyperoxia in neonatal rats. The results of $H \& E$ staining revealed that the alveolar space was thick and there were fewer alveoli at 1-3 days following incubation with $21 \%$ oxygen in the control group rats; however, the alveolar space was thinner after 7 days and after 14-21 days, the alveolar space became uniform, the pulmonary space was thinner, a spinous process appeared and the number of alveoli increased. Conversely, the hyperoxia-exposed neonatal rats displayed enlarged alveoli from 7 days and after 14-21 days, the number of alveoli significantly decreased, the alveolar septum thickened and the alveolar structure was simplified. In the control group, the thickness of the alveolar septum became thinner, while in the model group the alveolar septum became thicker, which was clearly different on days 7, 14 and 21 (Fig. 1A). The RAC was determined to evaluate the stage of lung development (Fig. S1A); the model group exhibited a significantly lower number of alveoli than the control group, beginning from 7 days following exposure to hyperoxia $(\mathrm{P}<0.05$. In addition, the thickness of the alveolar wall was measured by morphometry (Fig. S1B); the alveolar septa significantly increased at 7 days following exposure to hyperoxia in the model group $(\mathrm{P}<0.05)$.

Differentiation of AECIIs into AECIs is inhibited in rats with BPD. To investigate the differentiation of AECIIs into AECIs in the alveoli of rats with BPD, SPC (AECIIs marker) and AQP5 (AECIs marker) expression levels were detected by RT-qPCR and western blot analysis in the lung tissues of neonatal rats after $1,3,7,14$ and 21 days of exposure to hyperoxia or normal air. The upregulation of SPC mRNA expression was first detected on day 7 in the hyperoxic lung tissues compared to the normoxic group (Fig. 1B), followed 

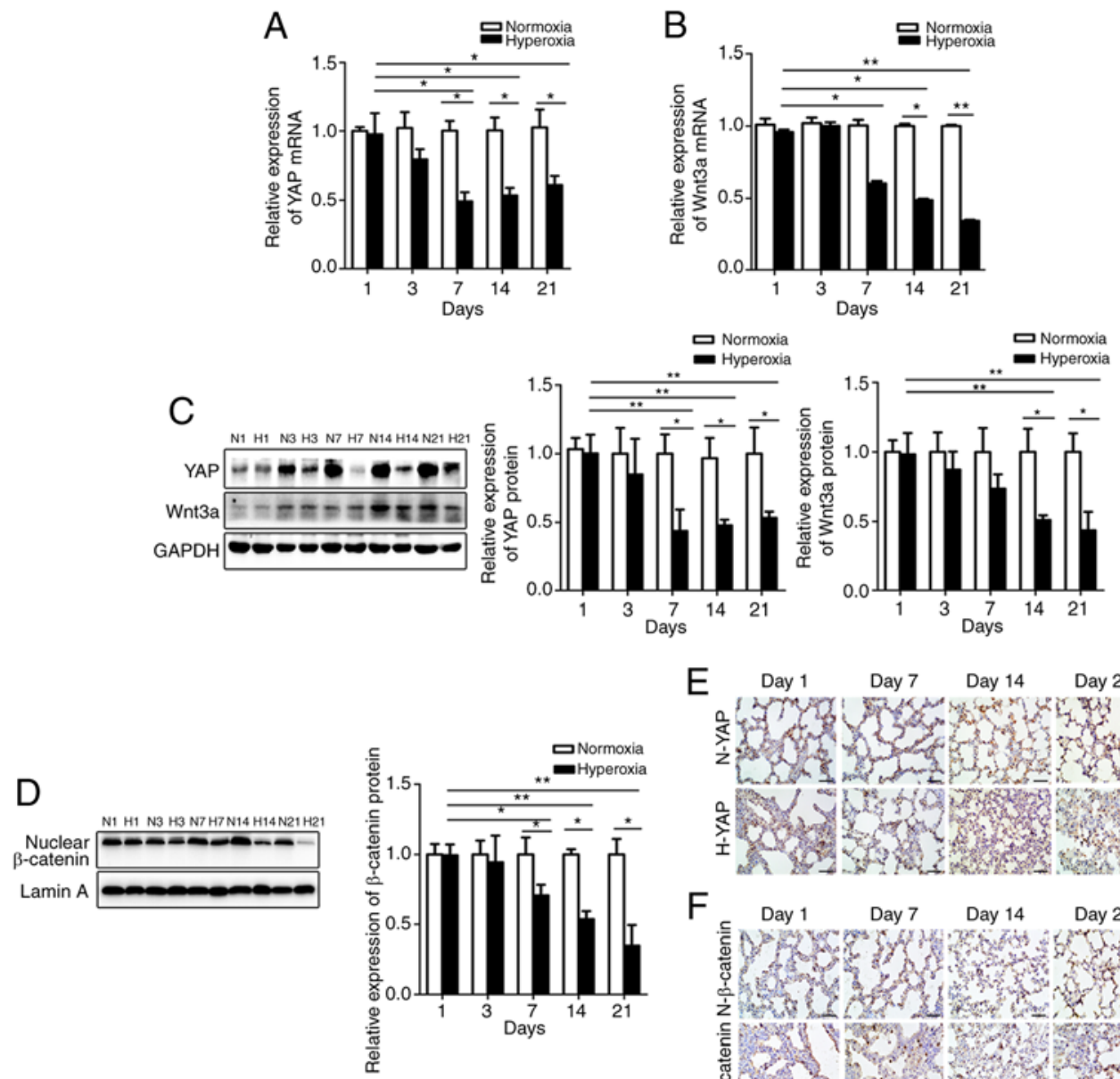

E $\begin{array}{llll}\text { Day } 1 & \text { Day } 7 & \text { Day } 14 & \text { Day } 21\end{array}$

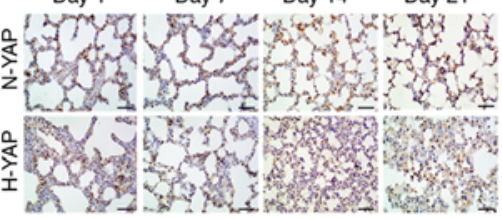

$\mathrm{F}$

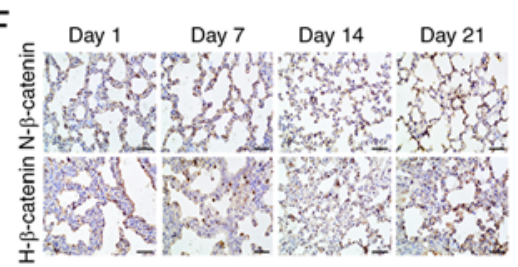

G SPC
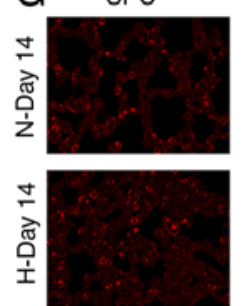

I SPC
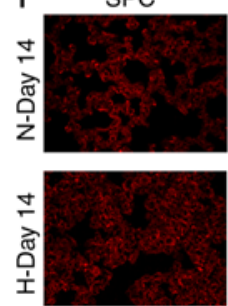

YAP
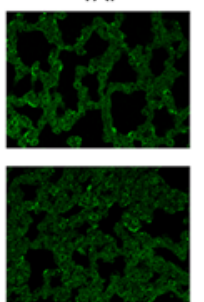

$\beta$-catenin
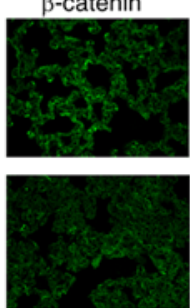

DAPI
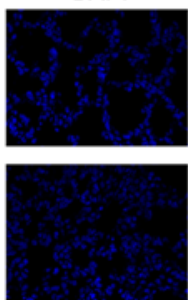

DAPI
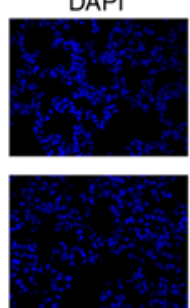

MERGE
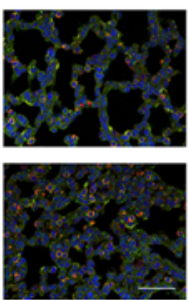

MERGE
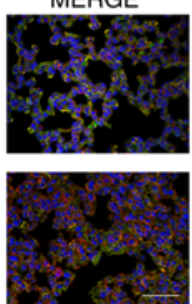

$\mathrm{H} \quad$ AQP5
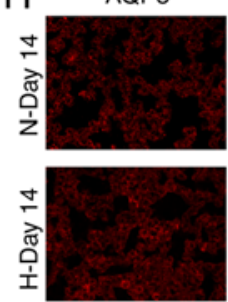

J $\beta$-catenin
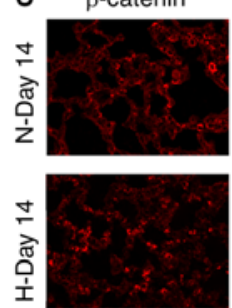

YAP
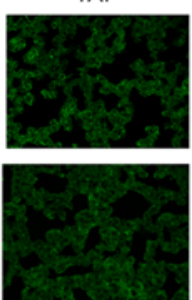

AQP5
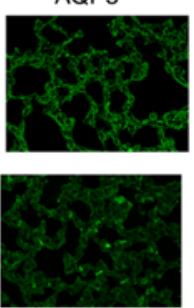

DAPI
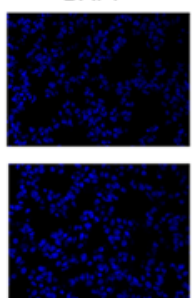

DAP
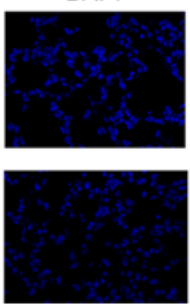

MERGE

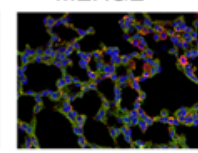

MERGE
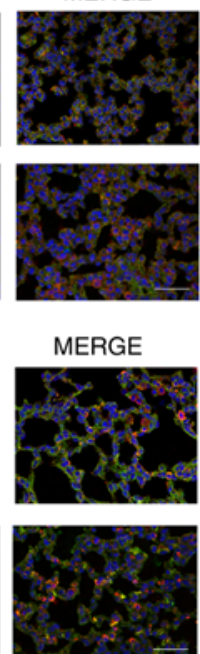

Figure 2. YAP, Wnt3a and $\beta$-catenin expression is decreased in lung tissue affected by BPD. (A) YAP mRNA and (C) protein expression in the lungs. (B) Wnt3a mRNA and (C) protein expression in the lungs. (D) $\beta$-catenin protein expression in lung nucleoli. Data are expressed as the means $\pm \mathrm{SD}(\mathrm{n}=6)$. ${ }^{*}<0.05,{ }^{* *} \mathrm{P}<0.01$. (E) YAP and (F) $\beta$-catenin expression in IHC-stained lung sections. Scale bar, $40 \mu \mathrm{m}$. Expression of (G) SPC and (G) YAP, as well as (H) AQP5 and YAP detected by double IF staining. Scale bar, $40 \mu \mathrm{m}$. (I and J) Expression of (I) SPC and $\beta$-catenin, as well as (J) AQP5 and $\beta$-catenin detected by double IF staining. Scale bar, $40 \mu \mathrm{m}$. N, normoxia-exposed rats; H, hyperoxia-exposed rats; SPC, surfactant protein C; AQP5, aquaporin 5; BPD, bronchopulmonary dysplasia.

by an increased expression over the subsequent days. In addition, AQP5 expression was markedly diminished compared to that in the control rats (Fig. 1C and D). Western blot analysis and RT-qPCR yielded similar results (Fig. 1D), indicating that differentiation was inhibited in the lungs of hyperoxia-exposed rats.
Expression of YAP, Wnt3a and nuclear $\beta$-catenin expression decreases in lungs affected by BPD. To determine whether YAP expression is affected in lungs of rats exposed to hyperoxia, YAP expression was verified in lung tissue at 1, 3, 7, 14 and 21 days in the experimental and control group neonatal rats by RT-qPCR, western blot analysis 

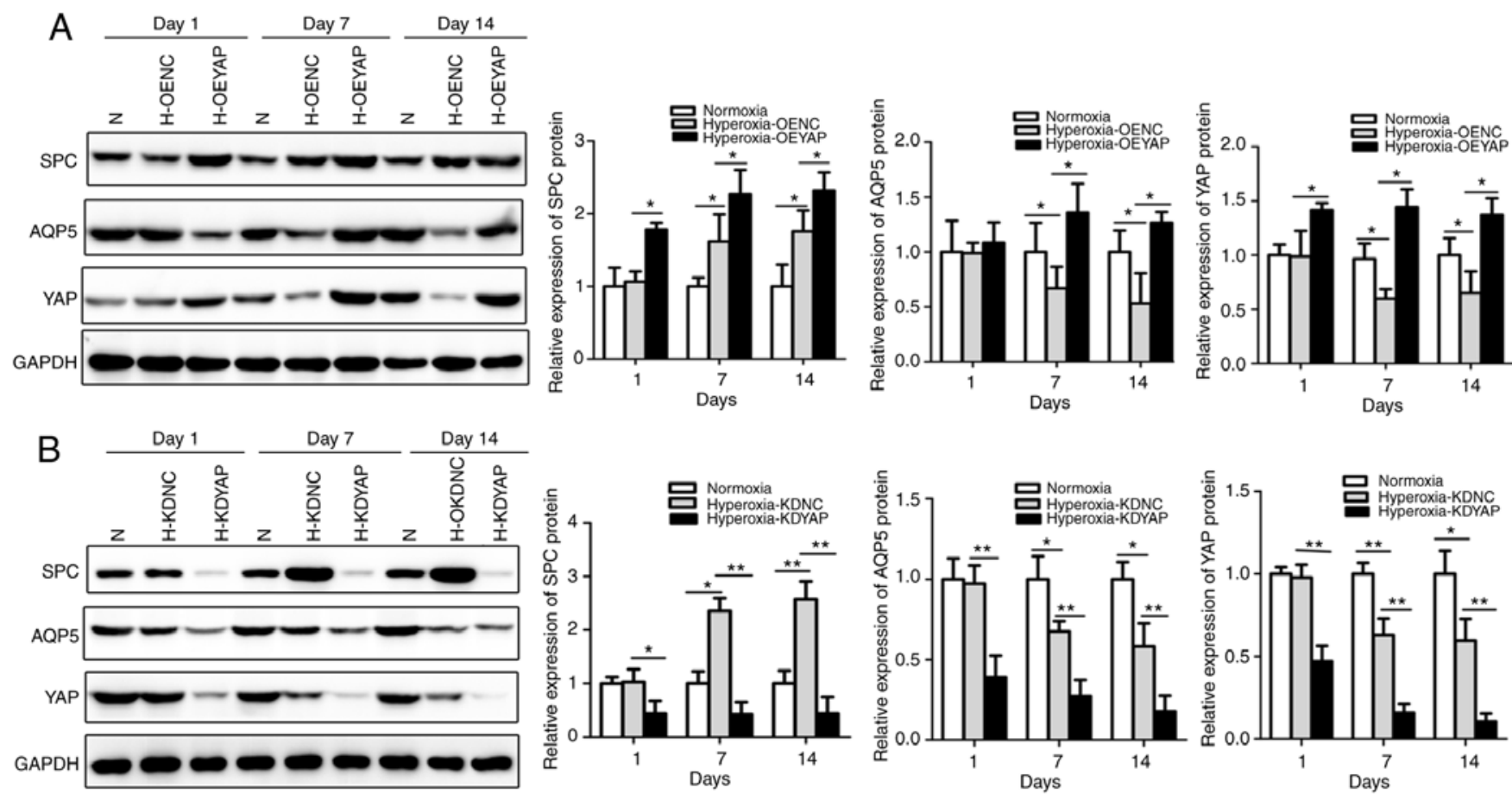

C
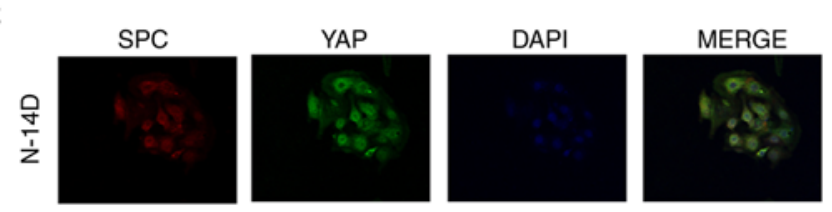

D
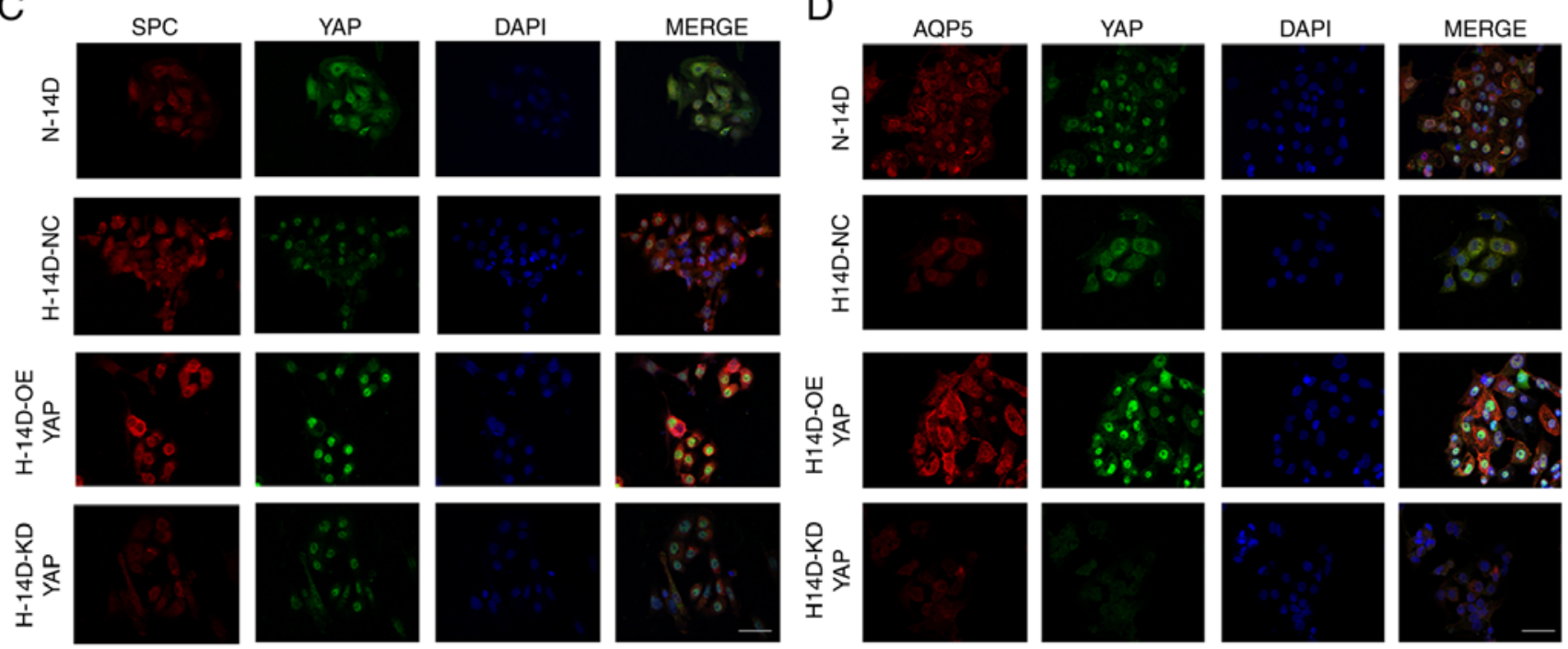

Figure 3. Effects of YAP on the proliferation and differentiation of AECIIs into AECIs. (A and B) SPC and AQP5 expression in OEYAP, KDYAP and corresponding control (OENC, KDNC) AECIIs detected by western blot analysis. Data are expressed as the means $\pm \mathrm{SD}(\mathrm{n}=3)$. ${ }^{*} \mathrm{P}<0.05$, ${ }^{* *} \mathrm{P}<0.01$. (C and $\left.\mathrm{D}\right) \mathrm{Double}$ IF staining for (C) SPC and YAP or (D) AQP5 and YAP in OEYAP, KDYAP and corresponding control (OENC, KDNC) AECIIs. Scale bar, $40 \mu \mathrm{m}$. N, normoxia-exposed rats; $\mathrm{H}$, hyperoxia-exposed rats; AECIIs and AECIs, alveolar epithelial type II and I cells; SPC, surfactant protein C; AQP5, aquaporin 5; $\mathrm{OE}$, overexpression; $\mathrm{NC}$, negative control; $\mathrm{KD}$, knockdown.

and IHC. The YAP mRNA levels began to decline on day 7 following exposure to hyperoxia compared with the normoxic group and continued to decrease thereafter (Fig. 2A), with western blot analysis, IHC and RT-qPCR yielding similar results (Fig. $2 \mathrm{C}$ and $\mathrm{E}$ ). In addition, Wnt3a expression (Fig. 2B and C) and nuclear $\beta$-catenin accumulation (Fig. 2D and F) were consistent with the results obtained for YAP expression.

To determine whether YAP and $\beta$-catenin are expressed on AECIIs or AECIs, double IF staining was performed for YAP/ $\beta$-catenin and SPC (AECII marker) or AQP5 (AECI marker). Although both YAP and $\beta$-catenin were expressed in the AECIIs and AECIs, their expression decreased in the
AECIIs and AECIs exposed to hyperoxia (Figs. 2G-J and S2). Therefore, YAP and $\beta$-catenin may be involved in the differentiation of AECIIs into AECIs.

Effects of YAP on the proliferation of AECIIs. The expression of SPC was detected in primary cultured AECIIs by IF (Fig. S3A), indicating successful cell isolation. Subsequently, YAP was overexpressed and knocked down (KD) in these cells using OEYAP plasmids and siRNAs, respectively. YAP mRNA and protein expression levels were higher in the OEYAP group than in the OENC group (Fig. S3B and D), and were significantly lower in the KDYAP group than in the KDNC group (Fig. S3C and D). 

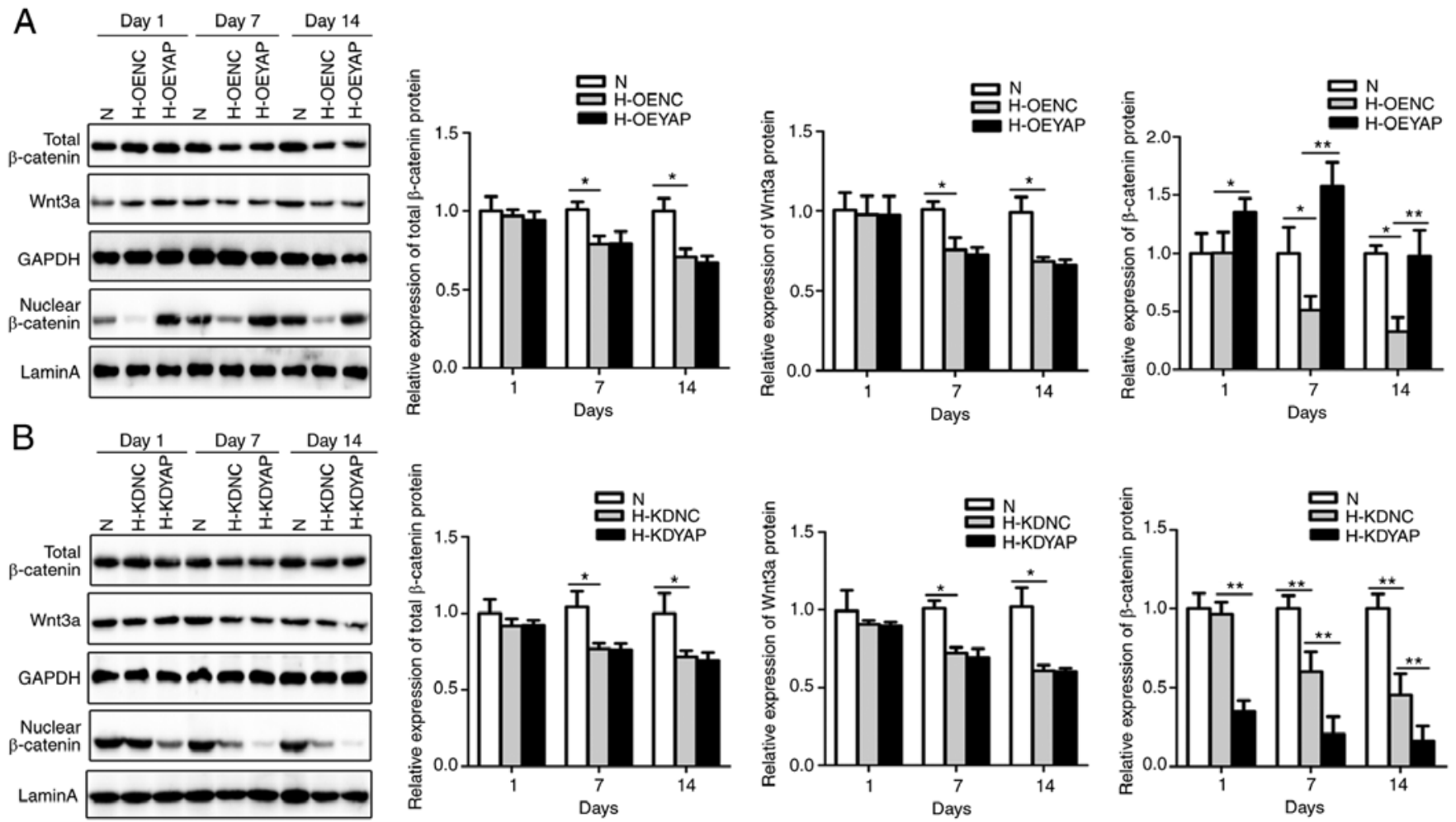

Figure 4. YAP overexpression and knockdown affects nuclear $\beta$-catenin, but not Wnt3a expression. (A and B) Expression of total and nuclear $\beta$-catenin in OEYAP, KDYAP and corresponding control (OENC, KDNC) AECIIs from rats exposed to hyperoxia for 1, 7 and 14 days, as determined by western blot analysis. Wnt3a expression in OEYAP, KDYAP and corresponding control (OENC, KDNC) AECIIs exposed to hyperoxia for 1, 7 and 14 days, as determined by western blot analysis. Data are expressed as the means $\pm \mathrm{SD}(\mathrm{n}=3) .{ }^{*} \mathrm{P}<0.05,{ }^{* *} \mathrm{P}<0.01$. N, normoxia-exposed rats; H, hyperoxia-exposed rats; AECIIs and AECIs, alveolar epithelial type II and I cells; OE, overexpression; NC, negative control; KD, knockdown.

YAP overexpression upregulated SPC expression in the OEYAP group compared with the OENC group (Fig. 3A), with stronger double IF staining observed for SPC and YAP in the OEYAP group (Fig. 3C). Therefore, YAP overexpression significantly promoted the proliferative capacity of the AECIIs.

Conversely, YAP knockdown (KDYAP) decreased SPC expression compared to that in the KDNC group (Fig. 3B), with weaker double IF staining observed for SPC and YAP in the KDYAP group (Fig. 3C). Thus, the genetic inhibition of YAP with siRNA appeared to significantly suppress the proliferation of AECIIs.

Effects of YAP on the differentiation of AECIIs into AECIs. YAP overexpression increased AQP5 protein expression compared to that in the OENC group (Fig. 3A) and produced stronger double IF staining for AQP5 and YAP (Fig. 3D), indicating that YAP overexpression promoted the differentiation of AECIIs into AECIs. Consistently, YAP knockdown decreased AQP5 protein expression compared to that in the KDNC group (Fig. 3B) and produced weaker double IF staining signals for AQP5 and YAP (Fig. 3D). Thus, YAP knockdown using siRNA against YAP may inhibit the differentiation of AECIIs into AECIs.

$Y A P$ affects the Wnt/ $\beta$-catenin signaling pathway via $\beta$-catenin, but not via Wnt $3 a$ in AECIIs. To investigate the mechanisms through which YAP affects the Wnt/ $\beta$-catenin signaling pathway, $\beta$-catenin expression was detected in
AECIIs in which YAP was overexpressed or knocked down. Total $\beta$-catenin expression differed slightly between the different groups (Fig. 4A and B), with higher nuclear $\beta$-catenin levels detected in the OEYAP group than in the OENC group (Fig. 4A), and lower $\beta$-catenin levels detected in the nuclei of the KDYAP group than in the KDNC group (Fig. 4B). However, YAP overexpression or knockdown did not significantly alter the Wnt3a levels, although it did activate nuclear $\beta$-catenin in the Wnt/ $\beta$-catenin signaling pathway (Fig. 4A and B). Taken together, these data indicate that YAP positively regulates Wnt/ $/ \beta$-catenin signaling by increasing the nuclear transfer of $\beta$-catenin without affecting Wnt3a expression in AECIIs.

Wnt3a affects the Wnt/ $\beta$-catenin signaling pathway via $\beta$-catenin, but not YAP in AECIIs. Wnt3a protein expression was markedly elevated in OEWnt3a AECIIs compared to that in the control AECIIs (Fig. S4A), while Wnt3a knockdown using siRNA decreased the Wnt3a mRNA and protein levels considerably compared to those in the KDNC group (Fig. S4B and C). Wnt3a overexpression increased SPC and AQP5 expression in the AECIIs compared with the OENC group (Fig. 5C), whereas Wnt3a knockdown decreased SPC and AQP5 expression (Fig. 5B). These results suggest that Wnt3a promotes the proliferation and differentiation of AECIIs into AECIs.

To confirm the mechanisms through which Wnt3a overexpression or knockdown affect nuclear $\beta$-catenin expression, $\beta$-catenin expression was detected in the nucleus. Wnt3a overexpression increased nuclear $\beta$-catenin levels 


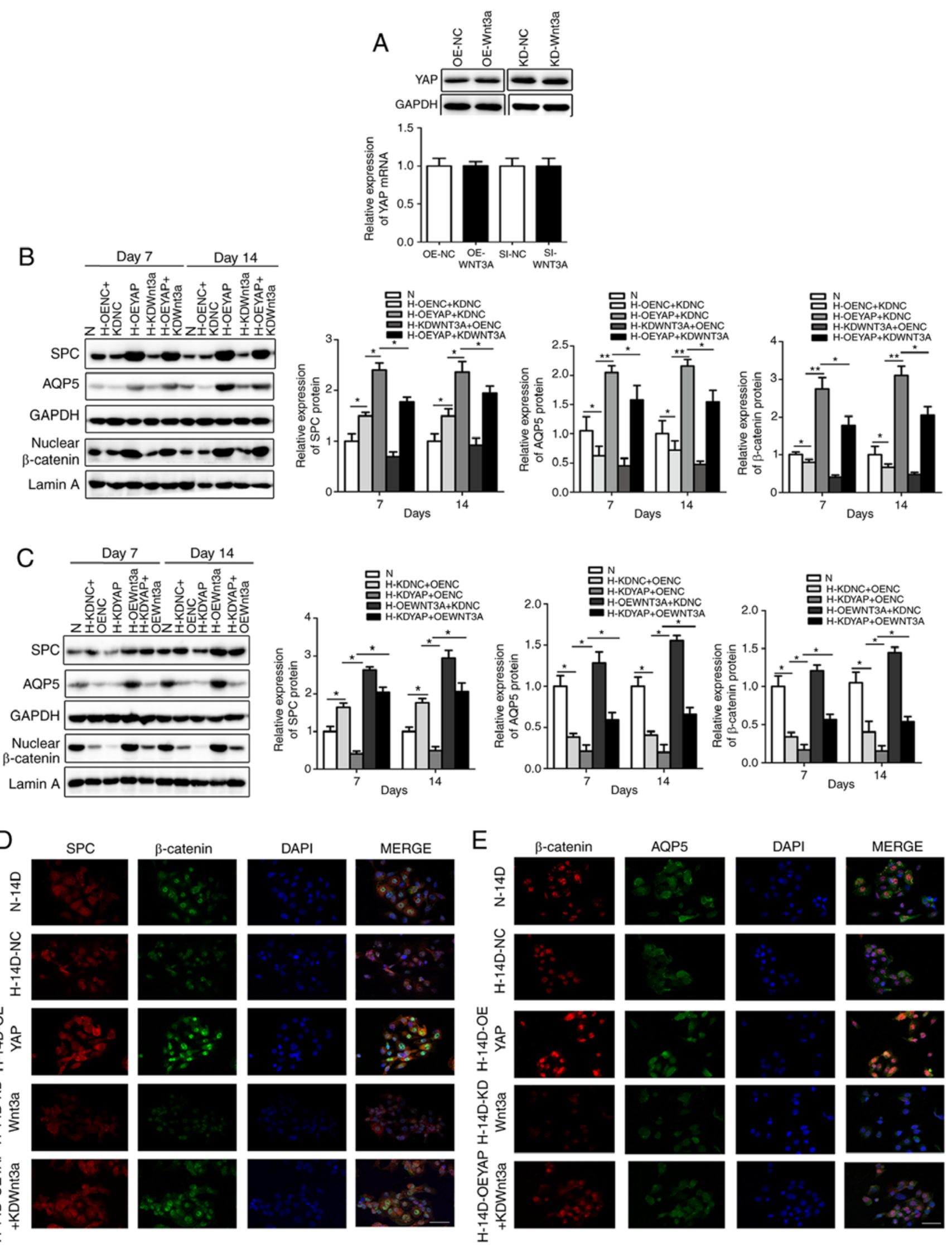

Figure 5. Continued.

compared to those in the OENC group (Fig. 5C), whereas Wnt3a knockdown decreased the $\beta$-catenin levels in the nucleus compared to those in the KDNC group (Fig. 5B).
However, Wnt3a overexpression or knockdown did not affect YAP expression (Fig. 5A), suggesting that Wnt3a positively regulates $\mathrm{Wnt} / \beta$-catenin signaling by increasing the nuclear 

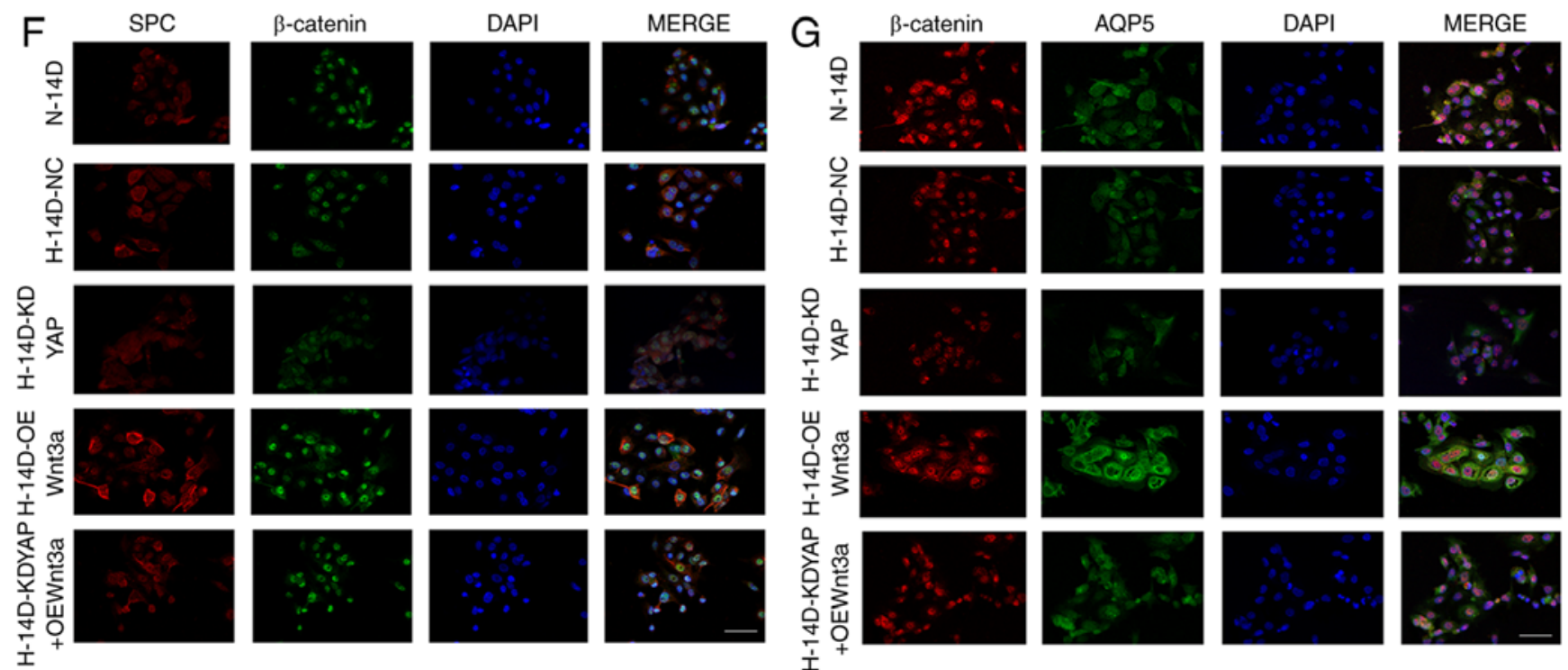

Figure 5. Wnt3a overexpression and knockdown reversed the effects of YAP on AECIIs proliferation and differentiation. (A) YAP expression in OEWnt3a, KDWnt3a and corresponding control (OENC, KDNC) AECIIs determined by western blot analysis. Data are expressed as the means \pm SD ( $n=3$ ). (B, D and E) SPC, AQP5 and nuclear $\beta$-catenin expression detected by (B) western blot analysis and double IF staining for (D) SPC and $\beta$-catenin or (E) AQP5 and $\beta$-catenin in OEYAP, KDWnt3a, OEYAP + KDWnt3a, and corresponding control (OENC, KDNC) AECIIs extracted from hyperoxia-exposed rats after 14 days. Data are expressed as the means $\pm \mathrm{SD}(\mathrm{n}=3)$. ${ }^{*} \mathrm{P}<0.05,{ }^{* *} \mathrm{P}<0.01$. Scale bar, $40 \mu \mathrm{m}$. (C, F and G) SPC, AQP5 and nuclear $\beta$-catenin expression detected by (C) western blot analysis and double IF staining for (F) SPC and $\beta$-catenin or (G) AQP5 and $\beta$-catenin in KDYAP, OEWnt3a, KDYAP + OEWnt3a and corresponding control (OENC, KDNC) AECIIs extracted from hyperoxia-exposed rats after 14 days. Data are expressed as the means \pm SD $(n=3)$. "P<0.05. Scale bar, $40 \mu \mathrm{m}$. N, normoxia-exposed rats; $\mathrm{H}$, hyperoxia-exposed rats; AECIIs and AECIs, alveolar epithelial type II and I cells; OE, overexpression; NC, negative control; KD, knockdown; SPC, surfactant protein C; AQP5, aquaporin 5.

transfer of $\beta$-catenin without affecting YAP expression in AECIIs.

Wnt3a overexpression and knockdown reverse the effects of YAP on the proliferation and differentiation of AECIIs. Since significant differences were observed following YAP overexpression or knockdown in the proliferation and differentiation of AECIIs extracted from rats exposed to hyperoxia for 7 and 14 days, these two time points for were selected subsequent functional experiments.

It was found that nuclear $\beta$-catenin expression was lower in the AECIIs in which YAP was overexpressed and Wnt3a was knocked down than in the OEYAP group (Fig. 5B), but was increased in AECIIs in which YAP was knocked down and Wnt3a was overexpressed compared with the KDYAP group (Fig. 5C). Thus, Wnt3a knockdown appears to reverse the effect of YAP overexpression on nuclear $\beta$-catenin, while Wnt3a overexpression reverses the suppressive effect of YAP knockdown on nuclear $\beta$-catenin.

Subsequently, functional experiments were performed to determine whether YAP regulates the proliferation and differentiation of AECIIs via $\mathrm{Wnt} / \beta$-catenin signaling. It was found that SPC and nuclear $\beta$-catenin expression and double IF staining were lower in the YAP-overexpressing AECIIs subjected to Wnt3a knockdown than in the OEYAP group (Fig. 5B and D). Moreover, SPC and nuclear $\beta$-catenin expression were higher in the AECIIs in which YAP was knocked down and in which Wnt3a was overexpressed than in the KDYAP group (Fig. 5C and F). The differentiation assay revealed that AQP5 and $\beta$-catenin expression and double IF staining were lower in the YAP-overexpressing AECIIs subjected to Wnt3a knockdown than in the OEYAP group (Fig. 5B and E), whereas AQP5 expression was higher in the AECIIs in which YAP was knocked down and subjected to Wnt3a overexpression than in the KDYAP group (Fig. 5C and G). Taken together, these results indicate that the effects of YAP overexpression or knockdown on the proliferation or differentiation of AECIIs can be partially reversed by inhibiting or activating the $\mathrm{Wnt} / \beta$-catenin signaling pathway.

\section{Discussion}

Recently, increasing attention has been paid to the regulation of stem cell biology by the Hippo-YAP/TAZ pathway; however, the effects of YAP on stem cell differentiation have not yet been fully defined (33-35). In the present study, it was found that YAP overexpression enhanced the number of AECIIs and AECIs, whereas its knockdown suppressed this process. Moreover, it was found that YAP regulated AECII proliferation and differentiation into AECIs in experimental BPD. It is generally believed that following lung injury, AECIIs can divide and restore the epithelium by differentiating into AECIs and replenishing the AECIIs population (36,37). Previous research has suggested that YAP regulates the proliferation of alveolar epithelial progenitors after acute lung injury (38); therefore, it was hypothesized that YAP may be a potential regulatory target in AECIIs and that its overexpression can facilitate lung regeneration or alveolar repair following hyperoxia-induced injury. Thus, inducing AECII proliferation and differentiation may serve as a novel therapeutic strategy for alveolar repair in BPD.

The in vitro and in vivo experiments performed in the present study demonstrated that the decreased YAP expression in hyperoxia-exposed lungs upregulated SPC and 
downregulated AQP5 expression; however, the number of AECIs decreased alongside the increase in AECIIs. This phenomenon may be explained by two factors: Firstly, other regulatory mechanisms may be involved in enhancing AECIIs proliferation; and secondly, the decreased differentiation of AECIIs into AECIs may lead to their accumulation in vivo and inhibit epithelial repair. Hou et al reported that the transdifferentiation of AECs was not suppressed, but rather was increased following exposure to hyperoxia by compensation; however, such repair during injury cannot offset pulmonary epithelial air exchange and barrier dysfunction caused by structural damage to AECs (39). In a word, the present study aimed to elucidate the pathological process of BPD from different perspectives and the possibility of different molecular expressions under different conditions. Depending on the cellular environment and stimuli, AECs respond to injury by undergoing different pathways. Different perspectives may lead to different outcomes, providing different insights for the treatment of BPD. In addition, it was also observed that the hyperoxia-induced inhibition of AECIIs differentiation into AECIs in vivo was accompanied by the downregulation of YAP expression; however, YAP overexpression contributed toward the proliferation and differentiation of AECIIs into AECIs in vitro. The different mechanisms employed by YAP in vivo and in vitro are likely due to alternative pathways that enhance the proliferation of AECIIs and inhibit YAP expression or involve decreased AP expression inhibiting the differentiation of AECIIs to AECIs, resulting in AECIIs aggregation in vivo.

YAP has also been shown to promote the proliferation of AECIIs in other models and induce their complete differentiation into AECIs (40). Moreover, previous studies have demonstrated that YAP is critical for the proliferation of AECIIs and differentiation into AECIs in response to mechanical tension in the adult lung following pneumonectomy (16). Another study reported that Hippo/YAP signaling regulates Ajuba in respiratory epithelial cells and controls progenitor cell proliferation and differentiation in developing and mature lungs (17). Furthermore, recent research has demonstrated that YAP adjusts AECII activities, including their proliferation, differentiation into AECIs, and inflammatory responses to balance both homeostasis and lung regeneration following lung injury induced by bacterial pneumonia (19). Conversely, some studies have suggested that LAS or MST deactivation leads to nuclear YAP accumulation, promotes the state of progenitor cells, and inhibits differentiation (41-45). Similarly, others have indicated that TAZ is necessary for the proliferation and differentiation of AECIIs into AECIs in vivo during alveolar repair after bleomycin injury; however, YAP is not involved in this process (20). The present study suggested that YAP contributed toward the proliferation and differentiation of AECIIs following hyperoxic lung injury. The inconsistent conclusions of these studies may be the result of factors, such as different stem cell sources, culture conditions, and YAP intervention models; however, they also suggest that the role of YAP in stem cell regulation is complicated and requires further research to be fully characterized.

The Wnt protein family controls a variety of developmental processes, including cell fate specification, proliferation, and migration. Therefore, Wnt signaling disorders during embryonic development can result in developmental defects (46).
Wnt signaling involves both a canonical and noncanonical pathway, which are known to be involved in lung development and diseases (25). In the present study, it was demonstrated that Wnt3a expression was downregulated in the lungs of rats exposed to hyperoxia and that Wnt3a overexpression and knockdown promoted and suppressed both SPC and AQP5 expression, respectively. In addition, it was found that Wnt3a regulated AECIIs proliferation and differentiation into AECIs in hyperoxia-induced BPD. Similarly, Wnt/ $\beta$-catenin has previously been reported to promote the differentiation of AECIIs into AECIs and an increased Wnt3a expression may contribute toward the secretion of pro-inflammatory factors in epithelial cells via the Wnt/ $\beta$-catenin pathway (47). However, the regulatory mechanisms of Wnt3a in AECIIs warrant further investigation.

Of note, the present study found that YAP overexpression or knockdown had no significant effect on total $\beta$-catenin and Wnt3a expression in vitro, but did affect nuclear $\beta$-catenin protein levels. To investigate the regulatory mechanisms of YAP in AECIIs, the present study examined the association between YAP and the Wnt/ $\beta$-catenin signaling pathway, finding that nuclear $\beta$-catenin levels, such as YAP expression, were lower in the lungs of rats exposed to hyperoxia. Therefore, it was hypothesized that the mechanism of $\beta$-catenin in AECIIs was similar to that of YAP. It was also found that YAP positively regulated the nuclear transfer of $\beta$-catenin, which was unaffected by Wnt3a in AECIIs, suggesting that YAP and Wnt signaling play essential roles in alveolar regeneration in AECIIs by mediating the switch to an AECIs fate after proliferation. Thus, the fine tuning of these two signaling pathways may help AECIIs to proliferate and differentiate sequentially, ultimately restoring the complex tissue architecture of the alveolar epithelium. The results are consistent with those of other research, in which YAP was reported to facilitate the nuclear translocation of $\beta$-catenin by promoting GSK-3b phosphorylation or reducing endogenous Dkk1 expression (48). Moreover, nuclear YAP/TAZ has been suggested to promote $\beta$-catenin nuclear translocation by mediating $\mathrm{T}$ cytokine-dependent transcription $(49,50)$. However, other studies have demonstrated that YAP inhibits Wnt signaling by inducing the endogenous expression of Dkk1, an inhibitory ligand of the Wnt pathway, thereby blocking Wnt/ $\beta$-catenin signaling (21). In addition, cytoplasmic YAP/TAZ can limit Wnt signaling activity by interacting with disheveled (DVL) or $\beta$-catenin $(14,29,51)$. In the absence of Wnt signaling, $\beta$-catenin remains phosphorylated, resulting in its degradation and the transcriptional inhibition of Wnt target genes (52). Therefore, nuclear YAP/TAZ can enhance Wnt signal transduction, whereas cytoplasmic YAP/TAZ inhibits Wnt signal transduction by activating the Hippo pathway (53). Although these studies were based on different cell models, their inconsistent conclusions suggest that YAP may regulate $\beta$-catenin via a variety of mechanisms.

To confirm whether Wnt3a regulates the proliferation and differentiation of AECIIs via YAP, Wnt3a interference assays were conducted. Wnt3a overexpression or knockdown reversed the effects of YAP on the proliferation and differentiation of AECIIs without affecting YAP expression, supporting the hypothesis that YAP promotes the proliferation and differentiation of AECIIs via nuclear $\beta$-catenin and not Wnt3a. Based on the effect of YAP on Wnt/ $\beta$-catenin 
signaling, it was confirmed that YAP partially regulated the proliferation and differentiation of AECIIs by activating the Wnt/ $\beta$-catenin signaling pathway; however, YAP must also be able to regulate this process via other pathways, which should be investigated in future studies. For instance, a recent study in a Pseudomonas aeruginosa acute infection model suggested that Dlk-1 downregulated Notch signaling to promote AECII-AECI differentiation (54). Thus, multiple developmental pathways, including Wnt, Hippo and Notch, likely work together to mediate tissue repair following alveolar injury. In particular, it has been suggested that alveolar epithelial cells from humans with pulmonary fibrosis display increased YAP activity and crosstalk with the mTOR/PI3K/AKT pathways (55). The current observations, as well as those of other mechanistic analyses (56), suggest that proper balance, sequencing, and cell-type specificity between the activity of the Wnt and Hippo pathways may be necessary to promote appropriate alveolar epithelial regeneration. YAP/TAZ is a transcriptional co-activating factor within and downstream of the Hippo signaling pathway that regulates multidirectional stem cell differentiation not only via Hippo signaling, but in coordination with a variety of pathways related to differentiation that form an interactive signaling network that jointly regulates the biological functions of stem cells. However, it remains unclear whether these reported mechanisms of YAP control the proliferation and differentiation of AECIIs since the role of YAP differs among organisms, making its regulatory mechanisms even more complex.

In conclusion, the present study demonstrated that both Wnt3a and YAP promote the proliferation and differentiation of AECIIs into AECIs during the pathogenesis of experimental BPD by activating the nuclear transfer of $\beta$-catenin. In addition, it was found that Wnt3a and YAP act independently without interacting with each other, Wnt3a knockdown appears to reverse the effect of YAP overexpression on nuclear $\beta$-catenin, while Wnt3a overexpression reverses the suppressive effect of YAP knockdown on nuclear $\beta$-catenin, and may play different roles in cell fate under different situations. Thus, further studies are required to elucidate their association in more detail. Taken together, the findings of the present study indicate that Wnt3a or YAP may serve as regulatory targets for improving the outcomes of BPD.

\section{Acknowledgements}

The authors are grateful to the Benxi Laboratory and the teachers at the laboratory for their assistance.

\section{Funding}

The present study was supported by grants from the National Natural Science Foundation of China (no. 81771621), the Key Research and Development Guidance Plan of Liaoning Province (no. 2019JH8/10300023), and the Natural Science Foundation of Liaoning Province (no. 20170541023).

\section{Availability of data and materials}

The datasets used and/or analyzed during the current study are available from the corresponding author on reasonable request.

\section{Authors' contributions}

WX and XJ designed the study. XJ wrote and revised the manuscript. XJ performed the research. BW, JH, LF and MY helped to design the overall study and analyze the data. All authors read and approved the final manuscript.

\section{Ethics approval and consent to participate}

All animal experiments were examined and approved by the Experimental Animal Ethics Committee of China Medical University.

\section{Patient consent for publication}

Not applicable.

\section{Competing interests}

The authors declare that they have no competing interests.

\section{References}

1. Li W, Chen Y,Zhang J, Hong L, Yuan N, Wang X and Lv H: IKBKE upregulation is positively associated with squamous cell carcinoma of the lung in vivo and malignant transformation of human bronchial epithelial cells in vitro. Med Sci Monit 21: 1577-1586, 2015.

2. O'Reilly M and Thébaud B: Animal models of bronchopulmonary dysplasia. The term rat models. Am J Physiol Lung Cell Mol Physiol 307: L948-L958, 2014.

3. Islam JY, Keller RL, Aschner JL, Hartert TV and Moore PE: Understanding the short- and long-term respiratory outcomes of prematurity and bronchopulmonary dysplasia. Am J Respir Crit Care Med 192: 134-156, 2015.

4. Lodha A, Seshia M, McMillan DD, Barrington K, Yang J, Lee SK Shah PS; Canadian Neonatal Network: Association of early caffeine administration and neonatal outcomes in very preterm neonates. JAMA Pediatr 169: 33-38, 2015.

5. Williams MC: Alveolar type I cells: Molecular phenotype and development. Annu Rev Physiol 65: 669-695, 2003.

6. Miyake Y, Kaise H, Isono KI, Koseki H, Kohno K and Tanaka M: Protective role of macrophages in noninflammatory lung injury caused by selective ablation of alveolar epithelial type II Cells. J Immunol 178: 5001-5009, 2007.

7. Mason RJ: Biology of alveolar type II cells. Respirology 11: S12-S15, 2006.

8. Shafa M, Ionescu LI, Vadivel A, Collins JJ, Xu L, Zhong S, Kang M, de Caen G, Daneshmand M, Shi J, et al: Human induced pluripotent stem cell-derived lung progenitor and alveolar epithelial cells attenuate hyperoxia-induced lung injury. Cytotherapy 20: 108-125, 2018.

9. Vega MA, Chupin C, Pascariu M, Privé A, Dagenais A, Berthiaume Y and Brochiero E: Dexamethasone fails to improve bleomycin-induced acute lung injury in mice. Physiol Rep 7: e14253, 2019.

10. Quantius J, Schmoldt C, Vazquez-Armendariz AI, Becker C, El Agha E, Wilhelm J, Morty RE, Vadász I, Mayer K, Gattenloehner S, et al: Influenza virus infects epithelial stem/progenitor cells of the distal lung: Impact on Fgfr2b-driven epithelial repair. PLoS Pathog 12: e1005544, 2016.

11. Yagi R, Chen LF, Shigesada K, Murakami Y and Ito Y: A WW domain-containing yes-associated protein (YAP) is a novel transcriptional co-activator. EMBO J 18: 2551-2562, 1999.

12. Meng Z, Moroishi T and Guan KL: Mechanisms of hippo pathway regulation. Genes Dev 30: 1-17, 2016.

13. Pan H, Xie Y, Zhang Z, Li K, Hu D, Zheng X, Fan Q and Tang T: YAP-Mediated mechanotransduction regulates osteogenic and adipogenic differentiation of BMSCs on hierarchical structure. Colloids Surf B Biointerfaces 152: 344-353, 2017.

14. Barry ER, Morikawa T, Butler BL, Shrestha K, de la Rosa R, Yan KS, Fuchs CS, Magness ST, Smits R, Ogino S, et al: Restriction of intestinal stem cell expansion and the regenerative response by YAP. Nature 493: 106-110, 2013. 
15. Sun C, De Mello V, Mohamed A, Quiroga HP, Garcia-Munoz A Al Bloshi A, Tremblay AM, von Kriegsheim A, Collie-Duguid E and Vargesson $\mathrm{N}$ : Common and distinctive functions of the hippo effectors taz and yap in skeletal muscle stem cell function. Stem Cells 35: 1958-1972, 2017.

16. Liu Z, Wu H, Jiang K, Wang Y, Zhang W, Chu Q, Li J, Huang H, Cai T, Ji H, et al: MAPK-Mediated YAP activation controls mechanical-tension-induced pulmonary alveolar regeneration. Cell Rep 16: 1810-1819, 2016

17. Lange AW, Sridharan A, Xu Y, Stripp BR, Perl AK and Whitsett JA: Hippo/Yap signaling controls epithelial progenitor cell proliferation and differentiation in the embryonic and adult lung. J Mol Cell Biol 7: 35-47, 2015.

18. Zhao B, Wei X, Li W, Udan RS, Yang Q, Kim J, Xie J, Ikenoue T, $\mathrm{Yu} \mathrm{J}, \mathrm{Li} \mathrm{L}$, et al: Inactivation of YAP oncoprotein by the hippo pathway is involved in cell contact inhibition and tissue growth control. Genes Dev 21: 2747-2761, 2007.

19. LaCanna R, Liccardo D, Zhang P, Tragesser L, Wang Y, Cao T, Chapman HA, Morrisey EE, Shen H, Koch WJ, et al: Yap/Taz regulate alveolar regeneration and resolution of lung inflammation. J Clin Invest 129: 2107-2122, 2019.

20. Sun T, Huang Z, Zhang H, Posner C, Jia G, Ramalingam TR, $\mathrm{Xu} \mathrm{M}$, Brightbill H, Egen JG, Dey A and Arron JR: TAZ is required for lung alveolar epithelial cell differentiation after injury. JCI Insight 5: e128674, 2019.

21. Seo E, Basu-Roy U, Gunaratne PH, Coarfa C, Lim DS, Basilico C and Mansukhani A: SOX2 regulates YAP1 to maintain stemness and determine cell fate in the osteo-adipo lineage. Cell Rep 3 . 2075-2087, 2013

22. Xu W, Xu B, Zhao Y, Yang N, Liu C, Wen G and Zhang B: Wnt5a reverses the inhibitory effect of hyperoxia on transdifferentiation of alveolar epithelial type II cells to type I cells. J Physiol Biochem 71: 823-838, 2015.

23. Xu W, Zhao Y, Zhang B, Xu B, Yang Y, Wang Y and Liu C: Wnt3a mediates the inhibitory effect of hyperoxia on the transdifferentiation of AECIIs to AECIs. J Histochem Cytochem 63 : 879-891, 2015.

24. Cohen JC, Larson JE, Killeen E, Love D and Takemaru K: CFTR and $\mathrm{Wnt} /$ beta-catenin signaling in lung development. BMC Dev Biol 8: 70, 2008

25. Freese JL, Pino D and Pleasure SJ: Wnt signaling in development and disease. Neurobiol Dis 38: 148-153, 2010.

26. Katoh M: WNT signaling in stem cell biology and regenerative medicine. Curr Drug Targets 9: 565-570, 2008

27. Aumiller V, Balsara N, Wilhelm J, Günther A and Königshoff M: WNT/ $\beta$-Catenin signaling induces IL-1 $\beta$ expression by alveolar epithelial cells in pulmonary fibrosis. Am J Respir Cell Mol Biol 49: 96-104, 2013

28. Jia L, Zhang Y, Ji Y, Xiong Y, Zhang W, Wen Y and Xu X: YAP balances the osteogenic and adipogenic differentiation of hPDLSCs in vitro partly through the Wnt/ $\beta$-catenin signaling pathway. Biochem Biophys Res Commun 518: 154-160, 2019

29. Imajo M, Miyatake $K$, Iimura $A$, Miyamoto $A$ and Nishida E: $A$ molecular mechanism that links Hippo signalling to the inhibition of Wnt/ $\beta$-catenin signalling. EMBO J 31: 1109-1122, 2012.

30. Sun H, Choo-Wing R, Fan J, Leng L, Syed MA, Hare AA, Jorgensen WL, Bucala R and Bhandari V: Small molecular modulation of macrophage migration inhibitory factor in the hyperoxia-induced mouse model of bronchopulmonary dysplasia. Respir Res 14: 27, 2013

31. Jakkula M, Le Cras TD, Gebb S, Hirth KP, Tuder RM, Voelkel NF and Abman SH: Inhibition of angiogenesis decreases alveolarization in the developing rat lung. Am J Physiol Lung Cell Mol Physiol 279: L600-L607, 2000.

32. Livak KJ and Schmittgen TD: Analysis of relative gene expression data using real-time quantitative PCR and the 2(-Delta Delta C(T)) method. Methods 25: 402-408, 2001.

33. Wang Y, Yu A and Yu FX: The Hippo pathway in tissue homeostasis and regeneration. Protein Cell 8: 349-359, 2017.

34. Cai J, Zhang N, Zheng Y, de Wilde RF, Maitra A and Pan D: The hippo signaling pathway restricts the oncogenic potential of an intestinal regeneration program. Genes Dev 24: 2383-2388, 2010

35. Grijalva JL, Huizenga M, Mueller K, Rodriguez S, Brazzo J, Camargo F, Sadri-Vakili G and Vakili K: Dynamic alterations in hippo signaling pathway and YAP activation during liver regeneration. Am J Physiol Gastrointest Liver Physiol 307: G196-G204, 2014.

36. Leslie CC, McCormick-Shannon K, Mason RJ and Shannon JM Proliferation of rat alveolar epithelial cells in low density primary culture. Am J Respir Cell Mol Biol 9: 64-72, 1993.
37. Evans MJ, Cabral LJ, Stephens RJ and Freeman G: Renewal of alveolar epithelium in the rat following exposure to NO2. Am J Pathol 70: 175-198, 1973.

38. Hu C, Sun J, Du J, Wen D, Lu H, Zhang H, Xue Y, Zhang A, Yang C, Zeng L and Jiang J: The hippo-YAP pathway regulates the proliferation of alveolar epithelial progenitors after acute lung injury. Cell Biol Int 43: 1174-1183, 2019.

39. Hou A, Fu J, Yang H, Zhu Y, Pan Y, Xu S and Xue X: Hyperoxia stimulates the transdifferentiation of type II alveolar epithelial cells in newborn rats. Am J Physiol Lung Cell Mol Physiol 308: L861-L872, 2015.

40. Zhou B, Flodby P, Luo J, Castillo DR, Liu Y, Yu FX, McConnell A, Varghese B, Li G, Chimge NO, et al: Claudin-18-Mediated YAP activity regulates lung stem and progenitor cell homeostasis and tumorigenesis. J Clin Invest 128: 970-984, 2018.

41. Zhao R, Fallon TR, Saladi SV, Pardo-Saganta A, Villoria J, Mou H, Vinarsky V, Gonzalez-Celeiro M, Nunna N, Hariri LP, et al: Yap tunes airway epithelial size and architecture by regulating the identity, maintenance, and self-renewal of stem cells. Dev Cell 30: 151-165, 2014

42. Lin C, Yao E and Chuang PT: A conserved MST1/2-YAP axis mediates hippo signaling during lung growth. Dev Biol 403: 101-113, 2015.

43. Chung C, Kim T, Kim M, Kim M, Song H, Kim TS, Seo E, Lee SH, Kim H, Kim SK, et al: Hippo-Foxa2 signaling pathway plays a role in peripheral lung maturation and surfactant homeostasis. Proc Natl Acad Sci USA 110: 7732-7737, 2013.

44. Szymaniak AD, Mahoney JE, Cardoso WV and Varelas X: Crumbs3-Mediated polarity directs airway epithelial cell fate through the hippo pathway effector yap. Dev Cell 34: 283-296, 2015.

45. Volckaert T, Yuan T, Chao CM, Bell H, Sitaula A, Szimmtenings L, El Agha E, Chanda D, Majka S, Bellusci S, et al: Fgf10-Hippo epithelial-mesenchymal crosstalk maintains and recruits lung basal stem cells. Dev Cell 43: 48-59, 2017.

46. Logan CY and Nusse R: The Wnt signaling pathway in development and disease. Annu Rev Cell Dev Biol 20: 781-810, 2004.

47. Wang Y, Huang C, Chintagari RN, Bhaskaran M, Weng T, Guo Y, Xiao X and Liu L: MiR-375 regulates rat alveolar epithelial cell trans-differentiation by inhibiting Wnt// -catenin pathway. Nucleic Acids Res 41: 3833-3844, 2013.

48. Yang B, Sun H, Song F, Yu M, Wu Y and Wang J: YAP1 negatively regulates chondrocyte differentiation partly by activating the $\beta$-catenin signaling pathway. Int J Biochem Cell Biol 87: 104-113, 2017.

49. Takahashi A, Tsutsumi R, Kikuchi I, Obuse C, Saito Y, Seidi A, Karisch R, Fernandez M, Cho T, Ohnishi N, et al: SHP2 tyrosine phosphatase converts parafibromin/Cdc73 from a tumor suppressor to an oncogenic driver. Mol Cell 43: 45-56, 2011

50. Tsutsumi R, Masoudi M, Takahashi A, Fujii Y, Hayashi T, Kikuchi I, Satou Y, Taira M and Hatakeyama M: YAP and TAZ, Hippo signaling targets, act as a rheostat for nuclear SHP2 function. Dev Cell 26: 658-665, 2013.

51. Varelas X, Miller BW, Sopko R, Song S, Gregorieff A, Fellouse FA, Sakuma R, Pawson T, Hunziker W, McNeill H, et al: The hippo pathway regulates Wnt/beta-catenin signaling. Dev Cell 18: 579-591, 2010.

52. Clevers H, Loh KM and Nusse R: Stem cell signaling. An integral program for tissue renewal and regeneration: Wnt signaling and stem cell control. Science 346: 1248012, 2014

53. Heallen T, Zhang M, Wang J, Bonilla-Claudio M, Klysik E, Johnson RL and Martin JF: Hippo pathway inhibits Wnt signaling to restrain cardiomyocyte proliferation and heart size. Science 332: 458-461, 2011.

54. Finn J, Sottoriva K, Pajcini KV, Kitajewski JK, Chen C, Zhang W, Malik AB and Liu Y: Dlk1-Mediated temporal regulation of notch signaling is required for differentiation of alveolar type II to type I cells during repair. Cell Rep 26: 2942-2954, 2019.

55. Gokey JJ, Sridharan A, Xu Y, Green J, Carraro G, Stripp BR, Perl AK and Whitsett JA: Active epithelial Hippo signaling in idiopathic pulmonary fibrosis. JCI Insight 3: e98738, 2018.

56. Aspal M and Zemans RL: Mechanisms of ATII-to-ATI Cell Differentiation during lung regeneration. Int J Mol Sci 21: 3188 , 2020. 